\title{
Deep Anterior Lamellar Keratoplasty as an Altermative Treatment on Granular Borneal Dystrophy
}

\section{JCR}

\author{
Faraby Martha, Bondan Harmani, Amir Shidik, Tjahiono D. Gondhowiardjo \\ Cornea and Refractive Surgery Division, Department of Ophthalmology, Faculty of Medicine \\ - University of Indonesia, Cipto Mangunkusumo Kirana Hospital, Jakarta, Indonesia.
}

\begin{abstract}
:
Introduction: Granular corneal dystrophies (GCD) is an autosomal dominant corneal disorder with bilateral visual disturbances. Deep anterior lamellar keratoplasty (DALK) was recently introduced as an alternative to keratoplasty. This case report mentions DALK as treatment modality for management of young patient with GCD. Case Report: A 24-year old Indonesian man presented with bilateral progressive visual loss since he was fifteen years of age. His bilateral best visual acuity was half meter finger counting. Slit lamp examination on both eyes revealed diffuse hazy corneas with bilateral opacities and multiple granular Bowman's plane and anterior half of stroma. The patient was diagnosed as having a granular corneal dystrophy of both eyes and DALK was performed with manual lamellar dissection technique on his right cornea. The same size of donor cornea was triphaned $1 / 2$ depth and put on top of host bare cornea, then nylon 10-0 was sutured. Two week after procedure, best corrected visual acuity in right eye improved to 6/40. Conclusion: DALK is an alternative procedure for management of patients with GCD whose corneal endothelium is not compromised.
\end{abstract}

Key words: Cornea, Corneal Dystrophies, Endothelium, Visual Aquity, Corneal Transplantation.

\section{Introduction}

Granular corneal dystrophy (GCD) is an autosomal dominant disorder characterized by small, breadcrumb-like, grayish-white, stromal opacities leading to varying degrees of visual disturbances [1-3]. Surgical intervention such as penetrating keratoplasty (PK), deep anterior lamellar keratoplasty (DALK), and phototherapeutic keratectomy are indicated in GCD patients with severe visual impairment or discomfort from recurrent corneal epithelial erosions [4]. Corneal dystrophy disorders treated with PK accounted for $4 \%$ to $24 \%$ of all corneal transplants [5]. Penetrating keratoplasty is the gold standard for the surgical treatment of most corneal pathologies, however many post-operative complications had been reported $[6,7]$.

Deep anterior lamellar keratoplasty (DALK) which was recently introduced as an alternative treatment to provide better visual outcomes, is

Corresponding Author: Dr. Tjahjono D. Gondhowiardjo

Email: tgondh@yahoo.com

Received: April 10, 2015 | Accepted: July 11, 2015 | Published Online: August 5, 2015

This is an Open Access article distributed under the terms of the Creative Commons Attribution License (creativecommons.org/licenses/by/3.0)

Conflict of interest: None declared | Source of funding: Nil | DOl: http://dx.doi.org/10.17659/01.2015.0086 
associated with lesser incidence of post-operative complications [6,7]. This procedure allows total stromal replacement of the recipient's cornea, leaving an intact recipient Descemet membrane and endothelium, to eliminate the risk of corneal donor rejection $[8,9]$. Deep anterior lamellar keratoplasty has abolished the risk for endothelial immune reactions in the avascular recipient bed to a great extent [10]. The objective of this case report is to describe the management of GCD with DALK procedure in young patient.

\section{Case Report}

A 24-year old Indonesian man came to our clinic with chief complaint of bilateral progressive visual loss since he was fifteen years old. He had no experiences of eye redness, floaters, photopsia, trauma, eye pain or watery eyes. There was no history of wearing spectacles, allergy, or other systemic illnesses. He denied having routine longterm medications or surgical history. He told that his young brother had similar symptoms. We examined his general health and found he was a healthy young man without any systemic diseases.

Eye examination showed his best corrected bilateral visual acuity (BCVA) was half meter finger counting. There was no nystagmus or any distubance of eyes movement in all direction. Intraocular pressure (IOP) was $20 \mathrm{mmHg}$ in his right eye and $19 \mathrm{mmHg}$ in his left eye (tonometry applanation Goldmann). The central corneal thickness of right eye was 742 and $703 \mu \mathrm{m}$ for his left eye. Corneal endothelial cell count was difficult to measure due to his corneal haziness.

Slit lamp examination revealed diffuse hazy corneas with bilateral opacities. The pattern of hazy cornea was multiple granular opacities located at the Bowman's plane and $1 / 2$ of corneal stroma depth on both eyes, but $2 \mathrm{~mm}$ of the peripheral cornea was still clear [Fig.1]. The funduscopy of both eyes was not appreciable due to corneal dystrophy. Examination of his brother's eyes also revealed mild stromal multiple granular opacities in both eyes. The patient was diagnosed with granular corneal dystrophy (GCD) of his both eyes and planned for bilateral DALK.

DALK was performed on his right eye in general anesthesia with manual lamellar dissection technique. Recipient corneal stroma was trephinated $1 / 2$ on depth cornea by Barron trephine, then manual dissection was performed to complete the procedure which corneal stroma was excised by lamellar surgical knives. Central stroma of host cornea was exposed $8.0 \mathrm{~mm}$ in diameter with $1 / 2$ corneal depth. Donor corneas were trephined using Barron donor punch with same size diameter as the recipient bed. The donor endothelium and Descemet membrane were dissected using toothed forceps. A $8.0 \mathrm{~mm}$ donor button cornea was placed in its proper position above an exposed stroma host cornea. After putting the donor cornea into host cornea, it was fixed by suturing the edge of donor to host cornea with the help of sixteen interrupted 10-0 nylon sutures. The procedure went unevently well. Unfortunetely, histopathologic examinations on an excised corneal recipient obtained from that procedure was not conducted. The patient was prescribed levofloxacin eyedrops
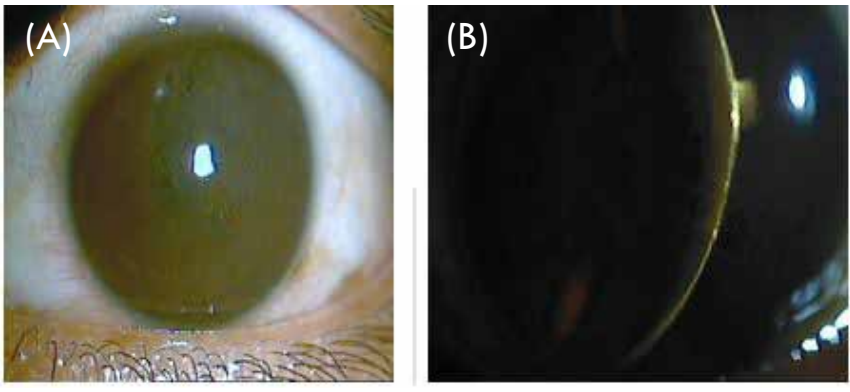

Fig.1:(A) Diffusely hazy corneas with opacities. (B) Multiple granular opacities located at the Bowman's plane and anterior half stroma. These findings were similar in both eyes. 
and corticosteroid eyedrops 6 times for right eye for 1 month.

Day one follow-up showed right eye visual acuity improving to $1 / 60$, graft edema, 16 healthy interupted stiches. The IOP was normal by using l-care tonometry. The anterior chamber was deep with minimal sel and flare, round iris pupil, clear lens, positive light reflect, but its cornea still looked moderately edematous. On one week follow-up, the visual acuity of his right eye was unchanged. Epithelial erosions with size of $3 \times 5 \mathrm{~mm}$ at superonasal corneal graft was seen [Fig.2]. The IOP of right eye was $10 \mathrm{mmHg}$ and the left eye was $20 \mathrm{mmHg}$. The deep anterior chamber with minimal sel and flare and mild oedema cornea was seen. Bandage lens was applied to corneal erosion, artificial tears and autologeus serum eye drops vitamin $C$ tablet were prescribed

Two weeks follow-up, the BCVA of the right eye increased to $6 / 40$ with correction of $S-8.00 \mathrm{D}$, $\mathrm{C}-10.5$ axis $90^{\circ}$. The keratometry reading of the right eye showed $\mathrm{KH} 51 \mathrm{D}-180^{\circ}$ and $\mathrm{KV} 40.50$ - $90^{\circ}$. The retinometry of right eye was $6 / 6$ and the left eye was $6 / 7.5$. The graft was clear with no epithelial erosions [Fig.3]. The IOP of right eye was $11 \mathrm{mmHg}$ and the left eye was $16 \mathrm{mmHg}$. Cornea was clear with decreased hyperemia in conjunctiva and normal anterior chamber. The bandage lens was removed from the right eye and eye treatment continued.

Three months follow up, showed BCVA at $6 / 40$ by using semihard contact lens. Corticosteroid and autologeus serum eye drops were stopped and treatment continued with artificial tears eye drops and oral vitamin $\mathrm{C}$.

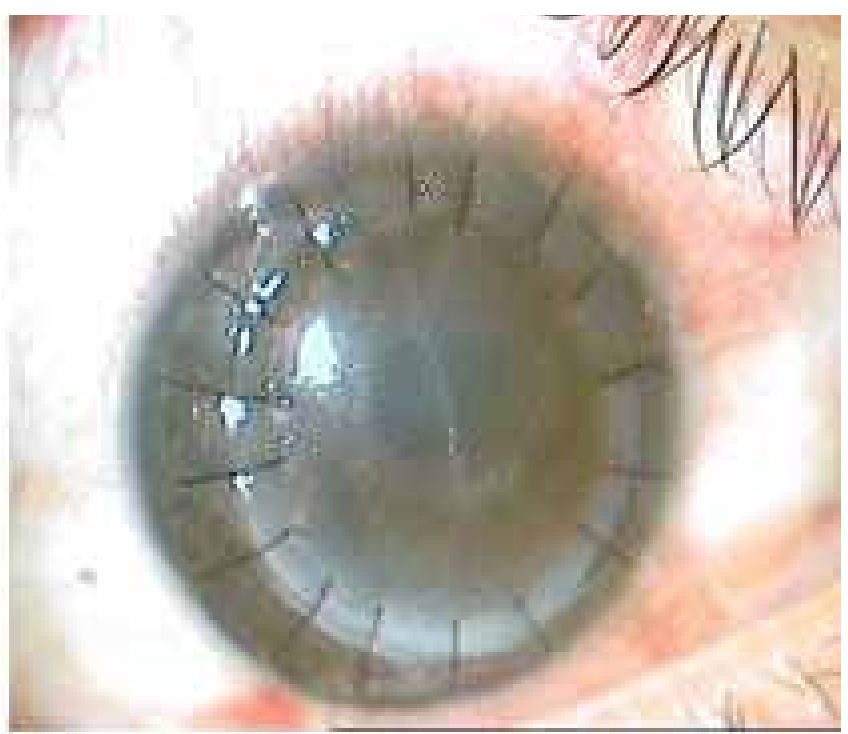

Fig.2: Slit lamp photography of the patient at 1 week follow up revealed minimally edema corneal graft with 16 interrupted stiches, and size $3 \times 5 \mathrm{~mm}$ central corneal graft erosion.

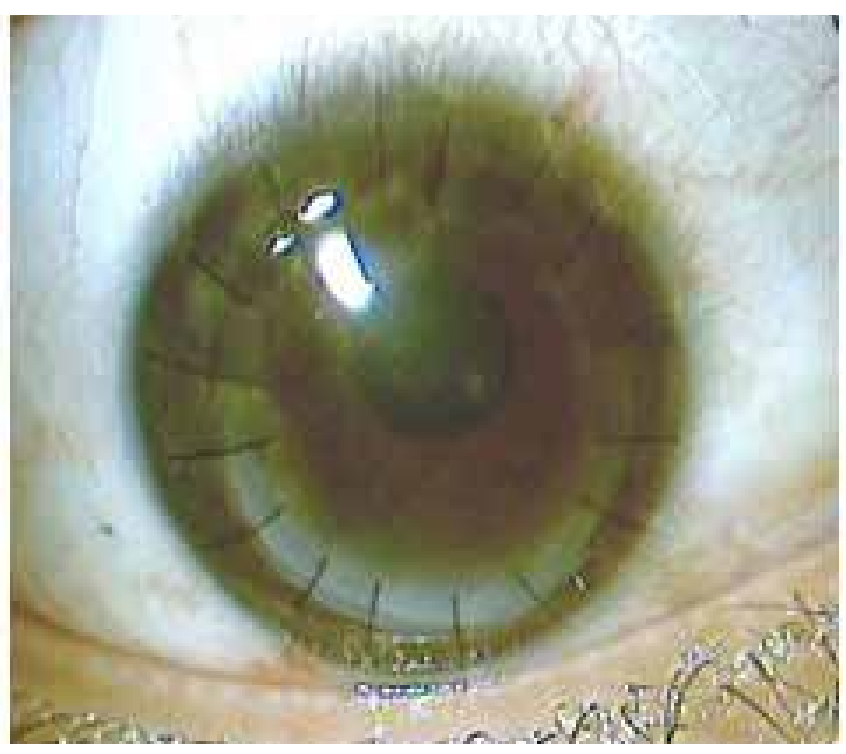

Fig.3: Slit lamp photography of the patient at 2 week follow up. Clear corneal graft with 16 interrupted stiches was seen. 


\section{Discussion}

We reported patient with bilateral granular corneal dystrophy (GCD) occupying $1 / 2$ depth corneal stromal layers. DALK surgery was performed with remarkable results in 3 month follow up. Many surgical interventions have been recommended in GCD patients with severe visual impairment, such as penetrating keratoplasty (PK), DALK, and phototherapeutic keratectomy (PTK), but preferred surgical option for management of $G C D$ has not been established yet $[11,12]$.

A healthy Descemet's membrane and corneal endothelium is the main criteria to decide DALK intervention [11]. In our case, DALK surgery had been applied since the GCD was located under Bowman's plane and anterior $1 / 2$ of stromal cornea. The advantages of DALK over PK includes decreased risk for endothelial graft rejection and late endothelial failure. It has been reported that endothelial cell attrition in DALK is less than PK, which subsequently reduces the risk of late graft failure [13]. In DALK loss of host endothelial cells is limited to $8 \%-15 \%$ during surgery [14]. As a lamellar procedure, DLK has all the advantages: avoiding most complications associated with "open-sky" surgery, lesser chances of anterior synechiae of iris, secondary glaucoma, and traumatic cataract [15]. Since the procedure is extraocular, DALK reduces the risk of intraocular surgical complications, such as choroidal hemorrhage, retinal detachment, cystoid macular edema and endophthalmitis [9]. Also DLK will maintain Descemet's membrane integrity, which is a major contributor to the cornea's tectonic strength and less traumatic dehiscence [16].

In our case, the patient still had clear lens, and healthy corneal cell endothelial count, therefore the DALK procedure has shown more advantages and less complications intraoperatively. As a young man in our case, who has abundant physical activities that might be increasing risk of trauma on his eyes and by doing DALK, corneal strength was very valuable for this young man patient. Another important benefit of DALK procedures is nylon 10-0 sutures can be removed earlier, although early reports suggested that sutures could be removed in DALK as early as 3 months postoperatively for a better wound healing [16]. Lyons et al. [12] compared the visual outcome, rate, and pattern of recurrence after DALK and PK for GCD. Although a good visual outcome was achieved earlier after DALK, the final visual acuities were marginally inferior to those after PK. This presents problems with interface irregularity, scarring and residual dystrophy noted in the deeper corneal layers or attributable to late interface opacification [17]. Cheng et al. [18] reported that BCVA after DALK was comparable to that in the PK group. Reduced steroids eye drop earlier may lead to a more prominent graft host junction and scarring in DALK. Regular and irregular astigmatism was common in DALK with similar problem on PK [6]. Irregular astigmatism occurs in 15\%-50\% of DALK, and patients with those problem can be corrected by rigid contact lenses but not spectacles $[6,19]$. Our patient was having high astigmatism after DALK, which could be corrected while using semi hard contact lens.

The most popular technique for deep anterior lamellar keratoplasty (DALK) is the "big bubble' (BB) technique wherein air is injected in the cornea to create a bubble that separates Descemet's membrane from the stroma. An attempt to create $B B$, additional manipulation may have a deleterious effect on the corneal endothelium [20]. Anwar et al. [21] pointed out that DALK technique even when done by a good-hand expert eye doctor, lamellar corneal dissection was still a difficult part to maneuver, which required a dry field and an good operating microscope to achieve uneven dissection plane. 
In this case, the DALK technique used was manual dissection, corneal depth dissection was controlled by operator with direct visual in microscope. In DALK, the recipient interface should be made as smooth as possible and ideally, it should consist only of Descemet's membrane and endothelium. Descemet's membrane detachment from the corneal stroma can be achieved by using air injection (the "big-bubble" technique), hydrodelamination through a sclerocorneal flap, or sodium hyaluronate injection [20,21]. Manual dissection can usually be performed to nearly of $90 \%$ corneal depth [14]. Borderie et al. [22] reported in DALK cases while was doing a manual dissection technique, the average thickness of the residual recipient stroma measured by OCT was 87 $\pm 26 \mu \mathrm{m}$ (range, 48-150 $\mu \mathrm{m}$ ). Manual dissection provides high failure to facilitates exposure of Descemet's membrane, higher risk of perforation, longer duration of the operation, and lower visual acuity achieved, but more simple, no new learning curve, and have higher survival of both the corneal endothelium and graft $[21,22]$.

Complications such as persistent epithelial defects and filamentary keratitis are more likely to develop [23]. Epithelial defects occured in our case, and the patient was managed well by bandage lens, and autologeus serum eyedrops. The use of $50 \%$ autologous serum eyedrops appears to be an efficacious medical treatment modality for corneal epithelial defects [24]. However, donor lamellar graft, recipient-bed interface complications such as interface space, Descemet's membrane wrinkling, and interface vascularization were uncommon [16]. Since endothelial immune graft rejection cannot occur after DALK, stromal rejection may occur in 1-2\% of DALK patients [25]. Although DALK procedure was inferior to $P K$ in more striving technique, more unique complication, poor BCVA and higher recurrence, but doing by handy surgeon will reduce rate of complication [26]. In this case the patient was a young man, therefore long graft survival was needed and long observation is necessary.

\section{Conclusion}

In our case DALK seems to be good alternative procedure for patients with granular corneal dystrophy on basis of endothelial cell loss, graft rejection, sutures management, corneal strength, and cataract complications.

\section{References}

1. Bron AJ. Genetics of the corneal dystrophies: what we have learned in the past twenty-five years. Cornea. 2000;19(5):699-711.

2. Aldave AJ, Rayner SA, King JA, Affeldt JA, Yellore VS. A unique corneal dystrophy of Bowman's layer and stroma associated with the Gly623Asp mutation in the transforming growth factor beta-induced (TGFBI) gene. Ophthalmology. 2005; $112(6): 1017-1022$.

3. Klintworth GK. Corneal dystrophies. Orphanet journal of rare diseases. 2009;4:7.

4. Cheng J, Qi X, Zhao J, Zhai H, Xie L. Comparison of penetrating keratoplasty and deep lamellar keratoplasty for macular corneal dystrophy and risk factors of recurrence. Ophthalmology. 2013;120(1):34-39.

5. Lang GK, Naumann GO. The frequency of corneal dystrophies requiring keratoplasty in Europe and the U.S.A. Cornea. 1987;6(3):209211.

6. Tan DT, Dart JK, Holland EJ, Kinoshita S. Corneal transplantation. Lancet. 201 2;379(9827):17491761.

7. Arenas E, Esquenazi S, Anwar M, Terry M. Lamellar corneal transplantation. Surv of Ophthalmol. 2012;57(6):510-529.

8. Mathur CV, Parihar BJKS, Srivastavac BVK, Avasthid MA. Clinical evaluation of Deep Anterior Lamellar Keratoplasty (DALK) for stromal corneal opacities. Medical Journal Armed Forces India. 
2013;69(1):21-26.

9. Luengo-Gimeno F, Tan DT, Mehta JS. Evolution of deep anterior lamellar keratoplasty (DALK). The Ocular Surface. 2011 ;9(2):98-110.

10. Steven P, Hos D, Heindl LM, Bock F, Cursiefen C. Immunreaktionen nach DMEK, DSAEK und DALK. [Immune reactions after DMEK, DSAEK and DALK]. Klinische Monatsblatter fur Augenheilkunde. 2013;230(5):494-499.

11. Shimazaki J, Shimmura S, Ishioka $M$, Tsubota $K$. Randomized clinical trial of deep lamellar keratoplasty vs penetrating keratoplasty. Am J Ophthalmol. 2002;134(2):159-165.

12. Lyons CJ, McCartney AC, Kirkness CM, Ficker LA, Steele AD, Rice NS. Granular corneal dystrophy. Visual results and pattern of recurrence after lamellar or penetrating keratoplasty. Ophthalmology. 1994;101(11):1812-1817.

13. Reddy JC, Rapuano CJ, Nagra PK, Hammersmith KM. Excimer laser phototherapeutic keratectomy in eyes with corneal stromal dystrophies with and without a corneal graft. Am J Ophthalmol. 2013;155(6): $1111-8$ e2.

14. Hannush SB. Deep anterior lamellar keratoplasty. In: Albert DM, Luceralli MJ, editors. Clinical atlas of procedures in ophthalmic and oculofacial surgery. 2nd ed. New York: Oxford university press; 2012. pp. 231-238.

15. Fournie P, Coullet J, Moalic S, Malecaze F, Chapotot E, Arne JL. Keratoplastie lamellaire anterieure profonde dans le traitement chirurgical du keratocone. Recul de plus d'un an [Deep anterior lamellar keratoplasty in the surgical treatment of keratoconus. A 1-year follow-up]. Journal francais d'ophtalmologie. 2006;29(6):602-613.

16. Reinhart WJ, Musch DC, Jacobs DS, Lee WB, Kaufman SC, Shtein RM. Deep anterior lamellar keratoplasty as an alternative to penetrating keratoplasty a report by the American Academy of Ophthalmology. Ophthalmology. $2011 ; 118(1): 209-218$.

17. Vajpayee RB, Tyagi J, Sharma N, Kumar N, Jhanji $V$, Titiyal JS. Deep anterior lamellar keratoplasty by big-bubble technique for treatment corneal stromal opacities. Am J Ophthalmol. 2007; 1 43(6):954-957.

18. Cheng YY, Visser N, Schouten JS, Wijdh RJ, Pels $E$, van Cleynenbreugel $H$, et al. Endothelial cell loss and visual outcome of deep anterior lamellar keratoplasty versus penetrating keratoplasty: a randomized multicenter clinical trial. Ophthalmology. 2011 ; 1 18(2):302-309.

19. Bhatt UK, Fares U, Rahman I, Said DG, Maharajan SV, Dua HS. Outcomes of deep anterior lamellar keratoplasty following successful and failed 'big bubble'. The British Journal of Ophthalmology. $2012 ; 96(4): 564-569$.

20. Huang $T$, Zhang $X$, Wang $Y$, Zhang $H$, Huand A, Gao N. Outcomes of deep anterior lamellar keratoplasty using the big-bubble technique in various corneal diseases. Am J Ophthalmol. 2012;154(2):282-289.

21. Anwar M, Teichmann KD. Big-bubble technique to bare Descemet's membrane in anterior lamellar keratoplasty. J Cataract Refractive Surg. 2002;28(3):398-403.

22. Borderie VM, Sandali O, Bullet J, Gaujoux T, Touzeau O, Laroche L. Long-term results of deep anterior lamellar versus penetrating keratoplasty. Ophthalmology. 2012;119(2):249-255.

23. Javadi MA, Feizi S, Javadi F, Kanavi MR, Ghasemi $\mathrm{H}$, Karimdizani $\mathrm{S}$, et al. Deep Anterior Lamellar Keratoplasty Using Fresh versus Cryopreserved Corneas. Ophthalmology. 2014;121:610-611.

24. Jeng BH, Dupps WJ, Jr. Autologous serum 50\% eyedrops in the treatment of persistent corneal epithelial defects. Cornea. 2009;28(10):11041108.

25. Kawashima $M$, Kawakita T, Den S, Shimmura S, Tsubota K, Shimazaki J. Comparison of deep lamellar keratoplasty and penetrating keratoplasty for lattice and macular corneal dystrophies. American journal of ophthalmology. 2006; 1 42(2):304-309.

26. Marcon AS, Cohen EJ, Rapuano CJ, Laibson PR. Recurrence of corneal stromal dystrophies after penetrating keratoplasty. Cornea. 2003;22(1):19-21. 